\title{
Diagnostic Difficulties in the Natural Rubber Latex Allergy
}

\author{
Mehmet Ünsel $\mathbb{D}$ \\ Department of Internal Medicine, Near East University Hospital, Division of Allergy and Immunology, Nicosia, Cyprus
}

ORCID iD of the author: U.M. 0000-0003-2152-3786.

Cite this article as: Mehmet U. Diagnostic Difficulties in the Natural Rubber Latex Allergy. Cyprus J Med Sci. 2021; 6(4): 345-348.

Natural rubber latex (NRL) allergy is a serious illness that presents itself with a spectrum of clinical signs and symptoms, including urticaria, allergic rhinitis, asthma, and anaphylaxis. NRL allergy is more commonly encountered among healthcare professionals and patients who undergo frequent surgical procedures because of sipina bifida or urogenital malformation. The ratio may rise to as high as $30 \%$ among children who have sipina bifida, while NRL allergy prevalence is just about $5 \%$ in healthcare professionals. Moreover, NRL allergy is the second most important cause of perioperative anaphylaxis. In routine practice, diagnosis of NRL allergy is based on specific lgE analysis and skin prick test. Nevertheless, not only is the diagnostic value of each one alone reaches $100 \%$, but also false positive test results are encountered. The precision of the diagnostic methods used for NRL allergy is important because false positive lgE results may negatively influence the patients' quality of life due to the stringent measures required to ensure latex-free environment. For this reason, the search for diagnostic methods, which would confer more sensitive and specific results for a precise diagnosis, has been intensified in the last two decades, and for this purpose, recently recombinant NRL allergens are being used for the diagnosis of NRL allergy. In this article, performances of old and new diagnostic methods used in NRL allergy and what we gained from recombinant NRL allergens have been discussed.

Keywords: Natural rubber latex allergy, diagnosis, skin prick test, in vitro tests, provocation test

\section{INTRODUCTION}

Natural rubber latex (NRL) is the sap of the Hevea brasiliensis tree. It is cheap and provides elasticity, durability, and protection to the materials for which it is produced; therefore, it is particularly used in the production of various medical equipment commonly used in the field of health care such as latex gloves, sphygmomanometer muff, branule, and hot water bag. Outside of medical sector, NRL is also used in the production of many goods, such as toys, balloons, feeding bottles, and preventatives, which are used commonly. Due to the widespread use of NRL in the field of health, primarily in healthcare professionals and in patients who undergo frequent surgical procedures connected to sipina bifida and urogenital malformation that cause intense NRL exposure, NRL allergy is more commonly encountered. According to the results of II epidemiological research that were conducted using skin prick test (SPT) and/or specific igE analysis, NRL allergy prevalence has been reported as $5.1 \%$ in healthcare professionals.' The ratio is higher and can even reach as high as $30 \%$ in children who have spina bifina. ${ }^{2}$ In general public, NRL allergy prevalence is under $1 \%{ }^{3}$

\section{NRL Allergens}

Up until today, 15 latex allergens have been identified (Hev b I-15). Out of these, Hev bl, 3, 5, and 6 are major, the rest are minor allergens. Hev I and 3 in children who have spina bifida and Hev b 5 and 6 in healthcare professionals are the major allergens that are responsible for this kind of allergy.'

\section{Clinical Manifestations of NRL Allergy}

NRL causes contact urticaria with skin contact, and allergic rhinitis and/or asthma via inhalation of airborne particles of the powdered latex gloves and anaphylaxis with mucosal (oral, vaginal, and gastrointestinal) or direct contact of inner organs during intraoperative period. The second most common cause of perioperative anaphylaxis is NRL allergy. ${ }^{4}$ For the patients who are diagnosed with NRL allergy, their contact with the entire group of products which contain NRL must be avoided for lifelong. Otolaryngological and gynecological examinations that involve direct mucosal contact and 
dental treatments, due to risk of anaphylaxis, must be performed with nonlatex gloves. For all surgical procedures of these patients, latex-free hospitals and operating rooms must be provided.

\section{Diagnosis of NRL Allergy}

In routine practice, diagnosis of NRL allergy is based on specific IgE analysis and SPT. Nevertheless, not only the diagnostic value of merely these methods alone is not $100 \%$, but also false positive test results are reported. 2,5 The precision of the diagnostic methods used for NRL allergy is of great importance because false positive IgE results may negatively influence the patients' quality of life due to the stringent measures required to ensure latex-free environment. That is why the diagnostic methods that would be more sensitive and specific for precise diagnosis have been intensified in the last two decades.

\section{Clinical History}

Although clinical history has great importance in diagnosis of NRL allergy, its specificity and predictive values alone are low. In a research studying the value of clinical history in diagnosis of NRL allergy with specific bronchial provocation test, $87 \%$ of sensitivity, $14 \%$ of specificity, $75 \%$ of positive predictive value (PPV), and $50 \%$ of negative predictive value (NPV) were determined. In the same study, when the positivity of both clinical history and SPT was accepted together as diagnostic criteria, sensitivity, specificity, and NPV increased (sensitivity of $100 \%$, specificity of $36 \%$, PPV of $76 \%$, and NPV of $71 \%$ ). ${ }^{6}$ Also, in the study conducted by Quirce et al. ${ }^{7}$ who utilized bronchial provocation test with NRL, diagnostic value of clinical history (sensitivity of $89 \%$, specificity of $50 \%$, PPV of $77 \%$, and NPV of $71 \%$ ) was found similar.

\section{Main Points}

- NRL allergy is a serious illness that presents with a spectrum of clinical signs, including urticaria, allergic rhinitis, asthma, and even anaphylaxis.

- It is more commonly reported among healthcare professionals and patients who undergo frequent surgery.

- The diagnosis of NRL allergy is based on specific lgE analysis and SPT in routine practice. Nevertheless, not only is the diagnostic value of each one alone reaches $100 \%$, but also false positive or negative results are encountered. Provocation tests should be used in patients whose diagnostic test results and clinical history are incompatible.

- Because of conjunctival, nasal, and bronchial provocation tests that have the risk of systemic allergic reaction, new in vitro tests including BAT and specific IgE analysis with recombinant NRL allergens have been started to use in the diagnosis of NRL allergy.

- SPT is still a more sensitive method than all the other in vitro tests in NRL allergy diagnosis. In clinical practice, the combined positivity of history and SPT seems enough in diagnosing NRL allergy most of the time. When clinical history and SPT are incompatible, specific lgE analysis or BAT with recombinant NRL allergens can give reliable results.

\section{Skin Prick Test}

The SPT sensitivity against NRL varies between 65 and $100 \%$. The difference in diagnostic criteria in NRL allergy as well as different allergen extract use might account for the wide-range in SPT sensitivity percentages. In 33 healthcare professionals with allergy history who were exposed to NRL, when NRLspecific IgE positivity (ImmunoCAP, Phadia, Uppsala, Sweden) was accepted as diagnosis criterion, the sensitivity, specificity, PPV, and NPV of SPT performed with five different NRL extracts ranged between 66 and $88 \%$, 9I and $95 \%, 75$ and $88 \%$, and 88 and $95 \%$, respectively. ${ }^{8}$ In 42 pediatric patients who displayed suspicion of allergy when the glove use test (provocation test) positivity was accepted as diagnostic criteria, the sensitivity and specificity of SPT performed with three different NRL extracts ranged between 65 and $96 \%$ and 88 and $94 \%$, respectively. ${ }^{9}$ Similarly, in two other studies in which bronchial provocation test with NRL was accepted as diagnostic criterion, the sensitivity and NPV of SPT were determined as 100\%, while specificity and PPV were determined as 20 vs $21 \%$ and 70 vs $74 \%$, respectively. ${ }^{6,7}$ As a result, when a credible testing such as the bronchial provocation test was accepted as diagnostic criterion, SPT sensitivity with NRL increased; however, specificity and PPV decreased. The reduced specificity and PPV of SPT performed with NRL point out that SPT may yield false positive results in some cases. Although not often, asymptomatic cases might be seen who show positive SPT with NRL. In such cases, provocation methods are warranted to obtain a precise diagnosis. Nevertheless, conducting a qualified provocation tests is not possible based on the facts that not only are these tests are cumbersome, but also test materials are not commercially available. In our previous nasal provocation test (NPT) study in which NRL extract $(500 \mu \mathrm{g} / \mathrm{mL} \mathrm{NRL}$ protein, ALK-Abello, Madrid, Spain) was used, NPT was found negative in two out of 26 patients who showed positive SPT with NRL. Those two nonhealthcare professional patients had polen allergy, yet they did not have NRL allergy history. Thereby, those two cases were accepted as false positive. It was suggested that this condition may have developed as a cross reaction due to pollen allergy. ${ }^{10}$

\section{In Vitro Tests}

Specific IgE analysis. Up until today, various in vitro methods (ImmunoCAP, DPC, AlaSTAT, and Hycor HyTEC) that research specific IgE presence to NRL were applied. The most commonly used one among those is ImmunoCAP (Uppsala, Sweden). Hamilton et al." compared three different latexspecific IgE analyses (ImmunoCAP, DPC AlaSTAT, and Hycor HyTEC). In this study, although the assay gave similar results, the best performance (sensitivity of $76.3 \%$, specificity of $96.7 \%$, PPV of $94.3 \%$, and NPV of $85 \%$ ) was obtained with immunoCAP when a positive SPT was accepted as the gold standard." Ownby et al. ${ }^{12}$ demonstrated that ImmunoCAP had a sensitivity of $79.5 \%$, specificity of $90.2 \%$, PPV of $91.7 \%$, and NPV of $76.4 \%$ when concomitant positivity of history and SPT was accepted as the gold standard. In our study, when taking the positivity of NPT and SPT together as the diagnostic criteria, we determined the sensitivity of immunoCAP $\mathrm{k} 82$ of $90 \%$, specificity of $72.2 \%$, NPV of $96.3 \%$, and PPV of $50 \%$. $^{13}$ These three studies exhibited that the diagnostic value of NRL-specific lgE analyze was lower compared to SPT. Particularly, in patients who have pollen allergy, probability of false positivity of ImmunoCAP k82 can rise up to $30 \% .^{10,13}$ In these patients, profilins and crossreactive carbohydrate determinants $(C C D)$ are responsible for 
nonclinical-related NRL specific IgE positivity. ${ }^{14}$ Hence, in patients allergic to pollen, due to high ratios of false positivity, ImmunoCAP k82 is not a convenient diagnostic method and must not be used in NRL prevalence studies.

Specific lgE analysis with NRL merely shows sensitization and cannot distinguish true NRL allergy or cross-reactivity. This distinction can be performed by means of specific lgE analyses with recombinant NRL allergens and CCD. Because of this, studies that research the diagnostic value of specific lgE analyses by using recombinant NRL allergens have accelerated in recent years. Recombinant NRL allergens and specific IgE analyses are studied with the methods of ImmunoCAP and Immuno Solid-phase allergen Chip (ISAC). ImmunoCAP carries out specific IgE analysis for each recombinant NRL allergen (Hevb I, 3, 5, 6.01, 6.02, 8, 9, and II), whereas ISAC (Phadia, Uppsala, Sweden) searches specific lgE response to 103 native/ recombinant allergens, in which five recombinant NRL allergens (Hevb I, 3, 5, 6.0l, and 8) are included. Vandenplas et al. ${ }^{15}$ studied responses of specific IgE (ImmunoCAP) to 12 recombinant NRL allergens (Hev b, I, 3, 5, 6.0I, 6.02, 7-12, and I5) in 82 patients who had occupational asthma and positive specific inhalation challenges (SIC) and SIC negative 25 cases encompassing the control group. In the patient group, Hev b 5 of $63 \%$, Hev b 6.01 of $78 \%$, and Hev b 6.02 of $78 \%$ were determined as positive, while in the control group, Hev b 5 of $12 \%$, Hev b 6.0 of $32 \%$, and Hev b 6.02 of $24 \%$ were determined as positive. ${ }^{15} \mathrm{OHt}$ et al. ${ }^{16}$ found diagnostic performance of ImmunoCAP and ISAC similar in 52 NRL allergic and 50 venom allergic patients (for Hev b 5 and 6.02 in ImmunoCAP sensitivity of 50 and $71 \%$, specificity of 100 and $100 \%$, PPV of 100 and $100 \%$, and NPV of 66 and $77 \%$, and in ISAC, sensitivity of 44 and $69 \%$, specificity of 100 and $100 \%$, PPV of 100 and $100 \%$, and NPV of 63 and $76 \%$, respectively). In Vandenplas' study and also in other similar studies, sensitivity and NPV of specific lgE with recombinant NRL allergens were found below $80 \%$.,15-18 Besides this, as seen in Vandenplas' study, specific IgE with major allergens such as Hev b 5 and 6 may be determined as positive also in healthy individuals. Following the determination of major recombinant allergens, recombinant allergen NRL Hev b 5 was added to this test kit (Hev b 5-amplified ImmunoCAP k82) to increase diagnostic value of ImmunoCAP k82. Seyfart et al. ${ }^{18}$ found sensitivity of Hev b 5-amplified ImmunoCAP k82 more superior than nonamplified ImmunoCAP k82 and ISAC (70, 62.5, and $55 \%$, respectively) in 40 patients who have NRL allergy. As seen in all the studies, sensitivity and NPV of specific lgE analyses (ImmunoCAP and ISAC) with recombinant allergens were determined lower compared to that of ImmunoCAP k82. Despite that, in individuals who have pollen allergy and especially multiple pollen allergy, ratio of false positivity of ImmunoCAP k82 is high. Because significant part (average 40\%) of NRL allergic patients is atopic and a significant part (21-60\%) of those is pollen sensitive, ImmunoCAP k82 is not a dependable diagnostic method in this patient group. ${ }^{13,14,19}$ Therefore, in patients with ImmunoCAP k82 positive who have not NRL allergy history, Hev b I, 3, 5, 6, 8 (NRL profilin), and CCD specific IgE must be searched in order to determine true NRL allergy or cross-reactivity. Without having major allergen positivity, Hev b 8 and/or CCD specific IgE being positive points out clinically irrelevant sensitization.

Basophil activation test. Given the fact that the sensitivity of specific IgE analyses performed with recombinant NRL allergens being lower compared to SPT, specific IgE analysis with
NRL not being superior to SPT and due to systemic allergic reaction risk that may develop in provocation tests, basophil activation test (BAT) has been used recently. BAT is a flow cytometric method on measurement of activation markers (CD63 and/or CD203c) that appear on the surface of basophil as a result of basophiles incubation with NRL in vitro environment. Sans et al. ${ }^{20}$ determined the sensitivity, specificity, PPV, and NPV of BAT carried out with NRL extract in 43 patients who had NRL allergy as being $90.5,100,100$, and $100 \%$, respectively. Same researcher, in his later study based on BAT performed with NRL extract and recombinant NRL allergens (Hev b 5, 6, and 6.0I) in 23 children who had NRL allergy showed that BAT performed with NRL extract yielded better results $(95.6 \%$ vs $86 \%$ ). In the same study when ImmunoCAP and BAT with recombinant NRL allergens used concomitantly, sensitivity (of 95.6\%) did not increase compared to the BAT performed with NRL extract. ${ }^{19}$ Later, the study enrolling 22 patients who had NRL allergy determined the sensitivity of the BAT with NRL extract below $80 \% .^{17}$ This study showed that BAT gave better results than specific lgE analysis (ISAC) with recombinant NRL allergens (Hev b I, 3, 5, and 6).

\section{Provocation Tests}

In cases when in vitro and in vivo (SPT) diagnostic methods do not be compatible with clinical history or in the setting of negative SPT in spite of positive in vitro test result, provocation tests should be used in order to differentiate asymptomatic sensitization from true NRL allergy. In provocation tests, skin and mucosas (sublingual, conjunctival, nasal, and bronchial) are exposed to NRL and manifesting symptoms and results are scored. Therefore, it is time consuming compared to in vitro tests and poses a risk of systemic allergic reaction. Besides, medications such as steroid, antihistamine, and broncodilatator must be stopped at certain periods prior to the provocation test. There are only a few studies about provocation tests due to difficulties of the procedure, risk of systemic allergic reaction, and absence of standardized commercial kits. ${ }^{10,21}$ We successfully applied NPT with NRL extract $\left(500 \mu \mathrm{g} \mathrm{mL}{ }^{-1}\right.$, ALK-Abello, Madrid, Spain) for the first time in 26 patients who had positive SPT with NRL, 35 atopic, and 30 healthy control subjects, and we did not observe systemic allergic reaction during NPT. Also, we compared the diagnostic performance of NPT versus glove usage test (GUT) with powdered latex gloves and showed that NPT is a more sensitive method compared with GUT (sensitivity of 96 vs $81 \%$, specificity of 100 vs $90 \%$, NPV of 98 vs $75 \%$, and PPV of 100 vs $93 \%$, respectively). Nucera et al. ${ }^{21}$ applied GUT, sublingual, conjunctival, nasal, and bronchial provocation tests with NRL extract ( $500 \mu \mathrm{g} \mathrm{mL}^{-1}$, ALK-Abello, Madrid, Spain) in 40 patients who had NRL allergy and 20 healthy control subjects. While sensitivity of oral and sublingual provocation test was determined as lower than $50 \%$, sensitivity of GUT, conjunctival, nasal, and bronchial provocation tests was determined above $70 \%$. Sensitivity of bronchial and NPT was even higher (76\% and $82 \%$, respectively). While the specificity and PPV of all the tests were 100\%, NPV of the NPT and bronchial provocation tests were determined higher $(75 \%$ and $70 \%$, respectively). In this study, systemic allergic reaction was not observed in any patient during provocation test. According to the results of both studies, NPT seems as a more reliable provocation method. When the difficulties are taken into consideration in other provocation test procedures, GUT whose sensitivity and specificity is close to bronchial and NPT appears as a useful and simple provocation method..$^{10}$ 
In summary, SPT is still a more sensitive method than all the other in vitro tests in NRL allergy diagnosis. In clinical practice, the combined positivity of history and SPT seems enough in diagnosing NRL allergy most of the time. Yet, in the setting of an absence of pollen allergy and when clinical history and SPT are incompatible, specific IgE analysis with NRL extract (ImmunoCAP) can give reliable results. In patients with pollen allergy, especially those presenting multiple pollen sensitization, specific IgE analysis (ImmunoCAP) with recombinant NRL allergens (Hev b I, 3, 5, 6, and 8) and CCD will be useful. Due to the fact that sensitivity of specific IgE analysis with recombinant NRL allergens is below $80 \%$ when specific IgE with recombinant NRL allergens is found to be negative, BAT known as a more sensitive method should be performed with NRL extract or recombinant allergens. Since recombinant NRL allergens and BAT have been used in the diagnosis of NRL allergy, conjunctival, nasal, and bronchial provocation tests have lost their importance in clinical practice. GUT, which is simple and does not require a device, can be helpful in determining true NRL allergic patients in clinical practice. In the future, nasal or bronchial provocation tests can only be used in scientific studies for the purpose of researching diagnostic performance of in vitro tests. Due to the probability of risk of systemic allergic reaction and difficulties in application, nasal and bronchial provocation tests might be replaced by BAT, which sensitivity, specificity, and predictive values are high. Besides, taking into consideration of ImmunoCAP k82 can give high false positive results in pollen sensitive patients in particular, SPT should be used in NRL allergy prevalence studies.

Peer-review: Externally peer-reviewed.

Author Contributions: Concept - U.M.; Resources - U.M.; Materials U.M.; Data Collection and/or Processing - U.M.; Analysis and/or Interpretation - U.M.; Literature Search - U.M.; Writing Manuscript - U.M.

Conflict of Interest: The authors have no conflicts of interest to declare.

Financial Disclosure: The authors declared that this study has received no financial support.

\section{REFERENCES}

I. Vandenplas O, Raulf M. Occupational latex allergy: The current state of affairs. Curr Allergy Asthma Rep. 2017;17(3):14. [CrossRef]

2. Pires G, Morais-Almeida M, Gaspar A, et al. Risk factors for latex sensitization in children with spina bifida. Allergol Immunopathol (Madr). 2002;30(I):5-13. [CrossRef]

3. Mari A, Scala E, D'Ambrosio C, Breiteneder H, Wagner S. Latex allergy within a cohort of not-at-risk subjects with respiratory symptoms: Prevalence of latex sensitization and assessment of diagnostic tools. Int Arch Allergy Immunol. 2007;143(2):I35-I43. [CrossRef]

4. Ebo DG, Fisher MM, Hagendorens MM, Bridts CH, Stevens WJ. Anaphylaxis during anaesthesia: Diagnostic approach. Allergy. 2007;62(5):47I-487. [CrossRef]

5. Rendeli C, Nucera E, Ausili E, et al. Latex sensitisation and allergy in children with myelomeningocele. Childs Nerv Syst. 2006;22(I):2832. [CrossRef]
6. Vandenplas O, Binard-Van Cangh F, Brumagne A, et al. Occupational asthma in symptomatic workers exposed to natural rubber latex: Evaluation of diagnostic procedures. J Allergy Clin Immunol. 2001;107(3):542-547. [CrossRef]

7. Quirce $S$, Swanson $M C$, Fernández-Nieto $M$, de las Heras $M$, Cuesta J, Sastre J. Quantified environmental challenge with absorbable dusting powder aerosol from natural rubber latex gloves. J Allergy Clin Immunol. 2003;III(4):788-794. [CrossRef]

8. van Kampen $V$, de Blay F, Folletti l, et al. Evaluation of commercial skin prick test solutions for selected occupational allergens. Allergy. 2013;68(5):651-658. [CrossRef]

9. Bernardini R, Pucci N, Azzari C, Novembre E, De Martino M, Milani $M$. Sensitivity and specificity of different skin prick tests with latex extracts in pediatric patients with suspected natural rubber latex allergy-A cohort study. Pediatr Allergy Immunol. 2008;19(4):315318. [CrossRef]

10. Unsel M, Mete N, Ardeniz O, et al. The importance of nasal provocation test in the diagnosis of natural rubber latex allergy. Allergy. 2009;64(6):862-867. [CrossRef]

II. Hamilton RG, Biagini RE, Krieg EF. Diagnostic performance of food and drug administration-cleared serologic assays for natural rubber latex-specific lgE antibody. The multi-center latex skin testing study task force. J Allergy Clin Immunol. 1999;103(5 Pt I):925930. [CrossRef]

12. Ownby DR, Magera B, Williams PB. A blinded, multi-center evaluation of two commercial in vitro tests for latex-specific lgE antibodies. Ann Allergy Asthma Immunol. 2000;84(2):193-196. [CrossRef]

13. Unsel M, Mete N, Ardeniz O, Sin A, Gülbahar O, Kokuludağ A. Diagnostic value of specific IgE analysis in latex allergy. Int Arch Allergy Immunol. 20I2;158(3):28I-287. [CrossRef]

14. Gürlek F, Ünsel $M$, Ardeniz $O$, et al. Misleading allergens in the diagnosis of latex allergy: Profilin and cross-reactive carbohydrate determinants. Int Arch Allergy Immunol. 2018;176(I):I-7. [CrossRef]

15. Vandenplas $O$, Froidure $A$, Meurer $U$, et al. The role of allergen components for the diagnosis of latex-induced occupational asthma. Allergy. 2016;7I(6):840-849. [CrossRef]

16. Ott H, Schröder C, Raulf-Heimsoth $M$, et al. Microarrays of recombinant Hevea brasiliensis proteins: A novel tool for the component-resolved diagnosis of natural rubber latex allergy. $J$ Investig Allergol Clin Immunol. 2010;20(2):129-138.

17. Ebo DG, Hagendorens MM, De Knop KJ, et al. Componentresolved diagnosis from latex allergy by microarray. Clin Exp Allergy. 2010;40(2):348-358. [CrossRef]

18. Seyfarth F, Schliemann S, Wiegand C, Hipler UC, Elsner P. Diagnostic value of the $\operatorname{ISAC}(B)$ allergy chip in detecting latex sensitizations. Int Arch Occup Environ Health. 2014;87(7):775-781. [CrossRef]

19. Sanz ML, García-Avilés MC, Tabar Al, et al. Basophil activation test and specific lgE measurements using a panel of recombinant natural rubber latex allergens to determine the latex allergen sensitization profile in children. Pediatr Allergy Immunol. 2006;17(2):148-156. [CrossRef]

20. Sanz ML, Gamboa PM, García-Avilés C, et al. Flow-cytometric cellular allergen stimulation test in latex allergy. Int Arch Allergy Immunol. 2003;I30(I):33-39. [CrossRef]

21. Nucera E, Pollastrini E, Sabato V, et al. Challenge tests in the diagnosis of latex allergy. Int $J$ Immunopathol Pharmacol. 2010;23(2):543-552. [CrossRef] 